\title{
Endoscopic nasal biliary drainage-guided approach after endoscopic ultrasound- guided hepaticojejunostomy: safe stent exchange technique
}

Endoscopic ultrasound (EUS)-guided biliary drainage is an alternative when endoscopic retrograde cholangiopancreatography (ERCP) has failed $[1,2]$. Covered self-expandable metal stents (CSEMS) or dedicated plastic stents are usually deployed $[3,4]$; however, especially when using a transhepatic approach, these stents have a risk of migrating into the abdominal cavity before fistula creation between the hepatic parenchyma and luminal wall. Plastic stents have a smaller diameter than CSEMS, so carry a greater risk of early stent occlusion if the bile is infected and viscous. If total gastrectomy is required, mucosa on the opposite side can become damaged when using CSEMSs. Therefore, an endoscopic nasobiliary drainage (ENBD) tube may be more effective, especially for EUS-guided hepaticojejunostomy (HJS). Because this is an external drainage technique, internal drainage using a plastic stent is however also required. Exchanging a stent from an ENBD tube to a plastic stent can be challenging because the lumen of the intestine is limited in size and the diameter of the fistula is small. We herein de- scribe safe stent exchange using an ENBguided approach.

An 81-year-old man underwent EUS-HJS for bile duct obstruction and acute cholangitis caused by gastric cancer peritonitis; however, the bile was extremely infected and viscous, so EUS-HJS was performed using an ENBD tube ( $\triangleright$ Fig. 1 a). Internal drainage was attempted once the cholangitis had resolved. The ENBD tube was repositioned from the nose to the mouth, as previously described [5]. A duodenoscope was then inserted over the ENBD tube into the intestine
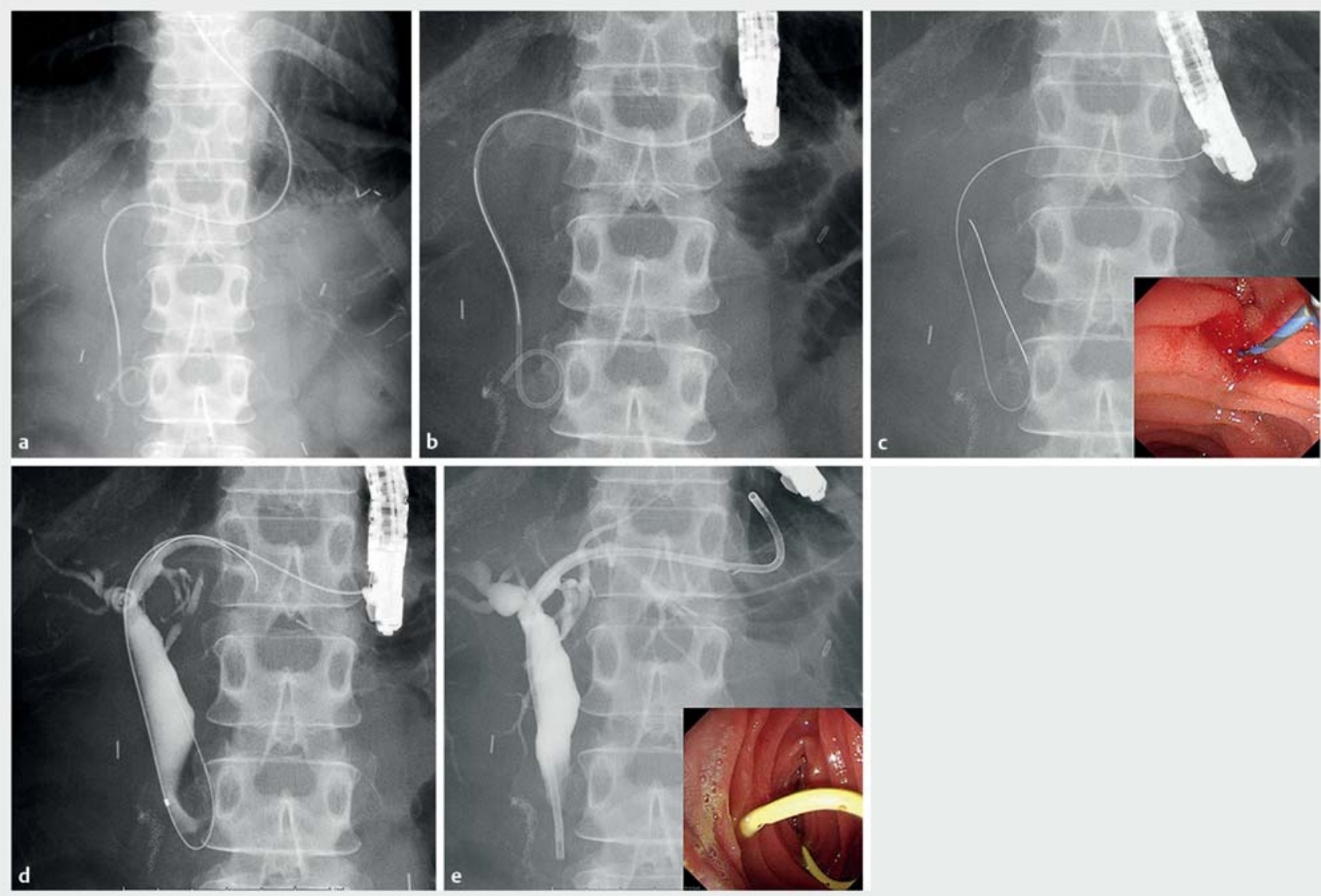

Fig. 1 Fluoroscopic images showing: a an endoscopic ultrasound-guided hepaticojejunostomy using an endoscopic nasobiliary drainage tube; b a duodenoscope inserted into the intestine over the endoscopic nasobiliary drainage tube; $\mathbf{c}$ the guidewire that was exchanged with the endoscopic nasobiliary drainage tube (inset: endoscopic view); $\mathbf{d}$ lower bile duct obstruction seen on the cholangiogram; e deployment of a dedicated plastic stent over the guidewire (inset: endoscopic view). 


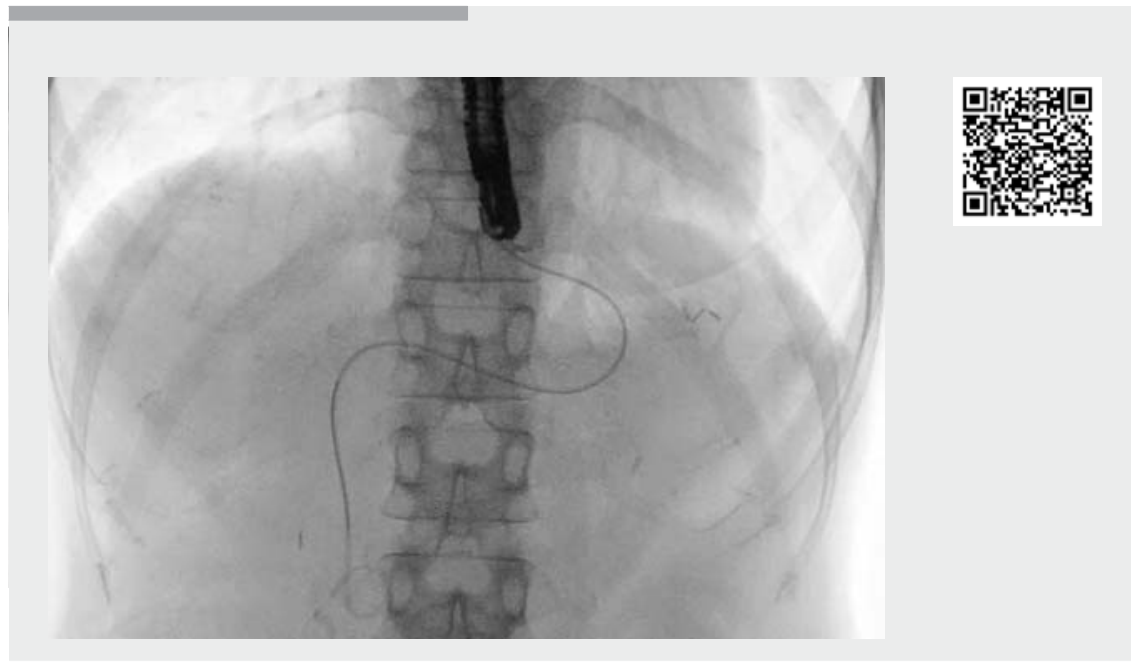

Video 1 A duodenoscope is inserted into the intestine over the endoscopic nasobiliary drainage tube, which is then exchanged for a guidewire. The endoscopic retrograde cholangiopancreatography catheter is inserted, contrast medium is injected. A dedicated plastic stent is deployed to treat the lower bile duct obstruction.

( Fig. 1 b). A guidewire was inserted into the ENBD tube, which was exchanged ( Fig.1 c). After cholangiography had been performed ( $\triangleright$ Fig. $\mathbf{1}$ d), a dedicated plastic stent was successfully deployed over the guidewire ( $\triangleright$ Fig. 1 e; $>$ Video 1 ). In conclusion, our technique using an ENBD tube is clinically useful and safe for biliary access during EUS-guided biliary drainage.

\section{Endoscopy_UCTN_Code_TTT_1AS_2AD}

\section{Competing interests}

The authors declare that they have no conflict of interest.

\section{The authors}

Takeshi Ogura, Saori Ueno, Yasukichi Tanaka, Azusa Hara, Kazuhide Higuchi

2nd Department of Internal Medicine, Osaka

Medical College, Osaka, Japan

\section{Corresponding author}

\section{Takeshi Ogura}

2nd Department of Internal Medicine, Osaka Medical College, 2-7 Daigakuchou,

Takatsukishi, Osaka 569-8686, Japan Fax: +81-72-6846532

oguratakeshi0411@yahoo.co.jp

\section{References}

[1] Isayama H, Nakai Y, Itoi T et al. Clinical practice guidelines for safe performance of endoscopic ultrasound/ultrasonographyguided biliary drainage: 2018. J Hepatobiliary Pancreat Sci 2019; 26: 249-269

[2] Hedjoudje A, Sportes A, Grabar S et al. Outcomes of endoscopic ultrasound-guided biliary drainage: A systematic review and meta-analysis. United European Gastroenterol J 2019; 7: 60-68

[3] Ogura T, Yamamoto K, Sano T et al. Stent length is impact factor associated with stent patency in endoscopic ultrasound-guided hepaticogastrostomy. J Gastroenterol Hepatol 2015; 30: 1748-1752
[4] Umeda J, Itoi T, Tsuchiya T et al. A newly designed plastic stent for EUS-guided hepaticogastrostomy: a prospective preliminary feasibility study (with video). Gastrointest Endosc 2015; 82: 390-396

[5] Kunishi Y, Kaneko T, Sugimori K. Introduction of roping techniques: a new induction method of ENBD tube to nostril using a guidewire (in Japanese). Gastroenterol Endosc 2012; 54: 2046-2047

\section{Bibliography}

DOI https://doi.org/10.1055/a-1113-7292

Published online: 21.2 .2020

Endoscopy 2020; 52: E310-E311

(c) Georg Thieme Verlag KG

Stuttgart · New York

ISSN 0013-726X

\section{ENDOSCOPY E-VIDEOS \\ https://eref.thieme.de/e-videos}

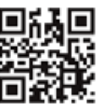

Endoscopy E-Videos is a free access online section, reporting on interesting cases and new

techniques in gastroenterological endoscopy. All papers include a high quality video and all contributions are freely accessible online.

This section has its own submission website at https://mc.manuscriptcentral.com/e-videos 